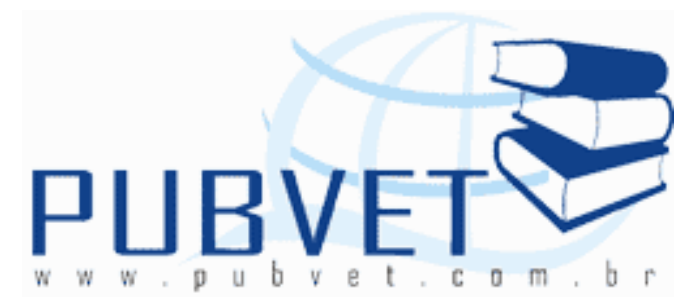

PUBVET, Publicações em Medicina Veterinária e Zootecnia.

\title{
Simulação do ganho em produtividade e associação entre características de crescimento e reprodutivas em um rebanho Nelore submetido à seleção
}

Edson Júnior Heitor de Paula ${ }^{1}$, Cláudio de Ulhôa Magnabosco ${ }^{2}$, Marco Antônio de Oliveira Viu ${ }^{3}$, Dyomar Toledo Lopes ${ }^{3}$, Luis Juliano Valerio Geron ${ }^{4}$, Eurico Lucas de Souza Neto ${ }^{4}$

${ }^{1}$ Professor da Universidade do Estado de Mato Grosso. edsonjr@unemat.br

2 Pesquisador da Embrapa Cerrados, bolsista CNPq, Planaltina, DF, Brasil. mclaudio@cpac.embrapa.br

${ }^{3}$ Professor da Universidade Federal de Goiás - Campus de Jataí, GO, Brasil.

${ }^{4}$ Professor da Universidade do Estado de Mato Grosso.

\section{Resumo}

O objetivo deste trabalho foi, por meio de modelo de simulação, verificar o resultado de vinte anos de seleção para crescimento e habilidade materna na produtividade e possível impacto sobre as características reprodutivas em um rebanho seleção Nelore puro de origem (PO), marca BRGN (Nelore BRGN), criado no bioma Cerrado. O modelo de simulação utilizado foi o DECI (Decision Evaluator for the Industry Cattle), onde os dados informados ao DECI buscaram refletir o mais fielmente possível, o sistema de produção do rebanho Nelore BRGN da Embrapa Cerrados. O modelo de simulação DECI foi eficaz em predizer a resposta à seleção genética para alta taxa de crescimento e 
habilidade materna, sinalizando melhoria direta nas características econômicas, por elevar o ganho de peso pré-desmame, o peso ajustado aos 205 dias e incrementar a produção de leite. A pequena diminuição na condição corporal e elevação do peso à maturidade das fêmeas não prejudicaram o desempenho reprodutivo. Sob as condições estudadas, a adoção das técnicas de manejo resultantes da simulação poderá aumentar as taxas produtivas do rebanho Nelore da Embrapa Cerrados sem prejuízo às características reprodutivas.

Palavras-chave: Cerrado, habilidade materna, Nelore, produção animal.

\title{
Simulation of the gain in productivity and association between growth and reproductive characteristics in a Nellore herd subjected to selection
}

\begin{abstract}
The aim of this study was, using a simulation model, to verify the result of twenty years of selection for maternal ability and growth in productivity and potential impacts on reproductive characteristics in a selection Nellore herd raised in the savannah biome. Data were reported to the DECI (Decision Evaluator for the Industry Cattle) simulation model reflecting the real situation of registered purebred Nellore herd of the Embrapa Cerrados (Nellore BRGN). The simulation model DECI was effective to predict the response to genetic selection for high growth rate and maternal ability, signaling direct improvement of economical characteristics, increasing pre-weaning weight gain and adjusted weight at 205 days and milk production. The slight decrease of females body condition and increase of maturity weight did not influence negatively the reproductive performance. Under the simulated conditions, the adoption of the management techniques resulted from the simulation may increase the productive rates of the Nellore Embrapa Cerrados herd without decrease performance on reproductive traits.
\end{abstract}

Keywords: savanna, maternal ability, Nellore bred, animal production. 


\section{INTRODUÇÃO}

A demanda mundial de carne bovina tem aumentado gradativamente com o aumento populacional e a melhoria da renda per capta facilitando o acesso a proteínas de origem animal. Para suprir tal demanda é necessário alto nível de produção, que só pode ser alcançado pelo melhoramento da composição genética dos animais, por meio da seleção e sistemas de acasalamento e melhoria nas condições ambientais da criação.

A definição dos objetivos de seleção é fundamental para o desenvolvimento dos programas de melhoramento genético, que de acordo com McMANUS et al. (2002), o processo de seleção deve objetivar a obtenção do genótipo bovino adequado ao sistema de produção de ciclo curto, sem aumentar as exigências nutricionais, alterando as formas das curvas de crescimento corporal e de desenvolvimento/maturação sexual, mantendo ou reduzindo o tamanho adulto, a necessidade de mantença e a idade de terminação ou acabamento de carcaças.

O melhoramento genético do material zebuíno, puro ou em cruzamento, deve ser monitorado de maneira sistemática, buscando identificar indivíduos que tenham maior velocidade de ganho em peso, precocidade de acabamento, habilidade maternal e eficiência reprodutiva dentro das variações existentes nos agroecosistemas pastoris (MAGNABOSCO, 2004).

Segundo MEIRELLES (2005) bovinos por possuírem intervalo de geração longo, retarda a visualização dos resultados da seleção, portanto tomadas de decisões para as características econômicas a serem selecionadas precisam ser bastante discutidas e pautadas no tempo, pois um critério de seleção escolhido erroneamente será identificado somente após anos de trabalho.

Dessa forma, a simulação computacional ou modelagem matemática vem de encontro a esse problema, servindo como ferramenta de alto desempenho e baixo custo, oferecendo maior eficiência na análise do impacto das mudanças ao longo do tempo nas diferentes estratégias de seleção utilizadas (TESS \& KOSTALD, 2000a,b). 
A simulação tem sido cada vez mais aceita e empregada como técnica que permite a analistas, dos mais diversos segmentos (administradores, engenheiros, biólogos, zootecnistas, entre outros), verificar ou encaminhar soluções, com a profundidade desejada, aos problemas com os quais lidam (PEGDEN, 1995).

Neste contexto, é considerada crescente a demanda mundial por sistemas de simulação. Porém, na literatura brasileira, existem poucos trabalhos que usaram modelos de simulação em sistemas de produção de gado de corte na região dos Cerrados (MAGNABOSCO et. al., 2006; PASCOA, et al., 2006, VIEIRA et al. 2006). Entretanto, em países de clima temperado, os modelos de simulação têm sido utilizados com sucesso para auxílio à pesquisa e na tomada de decisões em fazendas (SAINZ et al. 2000; TESS \& KOLSTAD, 2000a,b ; WILLIAMS et al., 2006).

O objetivo deste trabalho foi, verificar usando simulação, o impacto de vinte anos de seleção para crescimento e habilidade materna na produtividade e possíveis associações sobre as características reprodutivas em um rebanho seleção Nelore puro de origem (PO) criado no bioma Cerrado.

\section{MATERIAL E MÉTODOS}

O estudo foi conduzido utilizando dados provenientes da fazenda experimental da Embrapa Cerrados - Centro de Pesquisa Agropecuária dos Cerrados (CPAC), que está localizada no km 18 da BR 020, no município de Planaltina-DF, com altitude média de $1100 \mathrm{~m}$, latitude sul de $15^{\circ} 45^{\prime} \mathrm{e}$ longitude oeste de $47^{\circ} 25^{\prime}$ na qual são destinados 380 hectares de Cerrado para pastagens cultivadas. A temperatura média anual é de $20,5^{\circ} \mathrm{C}$ e o índice pluviométrico de $1300 \mathrm{~mm}$.

O modelo de simulação utilizado foi o DECI (Decision Evaluator for the Industry Cattle), desenvolvido pelos pesquisadores Charles Williams e Tom Jenkins do USDA - Meat Animal Research Center (MARC), Clay Center no estado de Nebraska-USA com o objetivo de dar suporte a tomadas de decisão 
estratégicas dos pecuaristas, permitindo então avaliar os impactos produtivos e econômicos que tais decisões trariam para a fazenda (WILLIAMS \& JENKINS, 2000).

O DECI, que foi desenvolvido em linguagem Fortran, é um modelo dinâmico de simulação que necessitou de várias pesquisas nas áreas de genética, nutrição, reprodução e manejo, para que fossem configuradas curvas de crescimento e parâmetros de desempenho dos animais para cada situação de manejo numa propriedade rural (WILLIAMS et al., 1995a,b,c).

O modelo DECI requer uma variedade de dados de entrada (inputs) que refletem diretamente o rebanho do sistema a ser simulado. Os inputs são informados ao programa por meio de quatro módulos principais, sendo eles: manejo, genética, alimentação e descarte. Dentro do módulo genética é informado o potencial genético para os critérios de seleção utilizados. Este potencial é descrito em três níveis categóricos: baixo, médio e alto para cada uma das variáveis de interesse econômico (nesse exemplo: habilidade maternal e potencial de crescimento).

Foram realizadas simulações com espaço temporal de vinte anos. Porém é importante observar que os primeiros cinco anos de simulação são descartados, para evitar a interferência dos valores iniciais sobre os resultados. Esta metodologia é adotada devido ao fato do modelo ser projetado para realizar observações no período de equilíbrio.

Nesta simulação utilizou-se a média dos dados reais de produção das safras de 1999 a 2006. O rebanho informado ao DECI era constituído de 229 fêmeas em reprodução, a estação de nascimento tinha início em julho e final em novembro, todas as crias eram pesadas ao nascer e a cada 90 dias, até completarem 24 meses de idade. A idade de desmama variou de 7 a 10 meses. A relação touro/vaca utilizada foi de $1 / 30$ e o tempo de permanência dos touros no rebanho foi de um ano. Todas as fêmeas em reprodução eram pesadas e passaram por diagnóstico de gestação na época da desmama, por meio do exame ginecológico. As novilhas iniciavam a estação reprodutiva com, pelo menos, 24 meses de idade. As vacas que permaneceram vazias ao final 
da estação de monta (EM) foram descartadas, e as novilhas mantidas, sendo estas colocadas novamente na EM no verão subseqüente. A EM tinha duração de 90 dias para as vacas (de 01/11 a 31/01) e 120 dias para as novilhas (01/10 a 31/01). A cada ano foram retidas 50 bezerras à desmama para reposição.

O rebanho Nelore, puro de origem (PO), marca BRGN da Embrapa Cerrados é participante do Programa de Melhoramento Genético da Raça Nelore - PMGRN, tendo como objetivo principal estabelecer um rebanho selecionado para a produção de animais geneticamente superiores para crescimento e habilidade materna, considerando o bioma de Cerrados. Utilizou-se no rebanho apenas touros Nelore PO que apresentaram alto potencial de crescimento e habilidade maternal quando comparados às médias nacionais disponibilizadas pelo PMGRN.

A base alimentar do rebanho da Embrapa Cerrados são pastagens cultivadas de braquiária (Brachiaria decumbens e brizantha cv. Xaraés), andropógon (Andropogon gayanus) e consorciadas de Brachiaria decumbens com estilosantes (Stylosanthes guyanenses cv. Mineirão). As informações referentes à oferta e qualidade de forragem foram baseadas em análises bromatológicas realizadas em amostras coletadas nas áreas de pastagem da Embrapa Cerrados. As categorias animais e período de pastejo das forrageiras disponíveis são apresentadas na Tabela 1.

$\mathrm{Na}$ Tabela 2 são apresentadas informações de proteína bruta (PB), nutrientes digestíveis totais (NDT) e disponibilidade potencial das forrageiras utilizadas durante o ano para a alimentação do rebanho. O programa de suplementação constituiu-se de suplemento protéico durante a seca (meses de junho a outubro) para todos os animais. Durante as águas (novembro a maio), os animais recebiam apenas sal mineralizado. O controle de consumo da mistura mineral foi mensal, obtido pela diferença de pesagem entre fornecido e sobras. A suplementação energética para bezerros foi feita pelo sistema creep-feeding. Na Tabela 3 estão sumarizadas as informações em relação ao 
período de consumo, quantidade, valor nutricional do suplemento e categoria animal suplementada.

Buscou-se, por meio de simulação, predizer o comportamento e a associação biológica de algumas características produtivas tais como: peso ajustado aos 205 dias (P205), ganho médio diário pré-desmame (GMD), peso à maturidade $(P M)$, condição corporal da vaca ao parto $(C C P)$, produção de leite $(\mathrm{PL})$, idade à puberdade (IP), idade ao primeiro parto (IPP), período de serviço (PS) e taxa de prenhez (TP).

Os resultados obtidos na simulação foram submetidos à análise de variância e posterior análise de regressão, utilizando-se os procedimentos GLM e REG do programa SAS (SAS, 1997).

TABELA 1 - Período de utilização das forrageiras disponíveis na Embrapa Cerrados de acordo com a categoria animal.

\section{Forrageiras}

\section{Brachiaria}

$\begin{array}{ccccc} & \text { Andropogon } & \text { Brachiaria } & \text { Brachiaria } & \text { decumbens + } \\ \text { Categoria } & \text { gayanus } & \text { decumbens } & \text { brizantha CV. } & \text { Stylosanthes } \\ \text { Animal } & & & \text { Xaraés } & \text { guyanenses } \\ & & & \text { Cv. Mineirão }\end{array}$

\begin{tabular}{|c|c|c|c|c|c|c|c|c|}
\hline & \multicolumn{8}{|c|}{ Época de pastejo } \\
\hline & Início & Fim & Início & Fim & Início & Fim & Início & Fim \\
\hline Vacas & $1 /$ nov & $31 /$ jan & $1 /$ jun & $31 /$ out & $1 /$ fev & $31 / \mathrm{mai}$ & - & - \\
\hline Bezerros & $1 /$ nov & 31/jan & - & - & $1 /$ fev & $31 / \mathrm{mai}$ & 1/abril & $31 /$ out \\
\hline Novilhas & $1 /$ nov & $31 /$ jan & - & - & $1 /$ fev & $1 /$ jun & 2/jun & $31 /$ out \\
\hline Touros & $1 /$ nov & 31/jan & $1 /$ mai & 31/out & $1 /$ fev & $30 / a b r$ & - & - \\
\hline
\end{tabular}


PAULA, E.J.H. et al. Simulação do ganho em produtividade e associação entre características de crescimento e reprodutivas em um rebanho Nelore submetido à seleção. PUBVET, Londrina, V. 6, N. 30, Ed. 217, Art. 1446, 2012.

TABELA 2 - Proteína bruta (PB), Nutrientes Digestíveis Totais (NDT) e disponibilidade potencial das forrageiras disponíveis para o rebanho Nelore da Embrapa Cerrados.

\begin{tabular}{|c|c|c|c|c|c|c|c|c|c|c|c|c|c|c|}
\hline \multirow{2}{*}{ tens } & \multicolumn{12}{|c|}{ Meses } & \multirow{2}{*}{\multicolumn{2}{|c|}{ Forrageira }} \\
\hline & Jan & Fev & Mar & Abr & Mai & Jun & Jul & Ago & Set & Out & Nov & Dez & & \\
\hline Proteína Bruta & 07 & 1. & 1 & 0 & 0 & 60 & 56 & 17 & 16 & 10 & 60 & & \multirow{4}{*}{\multicolumn{2}{|c|}{ 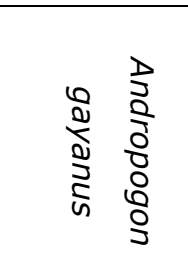 }} \\
\hline$\%$ da MS & & & & & & & & & & & & & & \\
\hline NDT & 59,0 & 60,2 & 59,0 & 55,0 & 53,0 & 50,0 & 48,1 & 45,1 & 45,0 & 48,1 & 52,0 & 56,0 & & \\
\hline Disponibilidade & 22,5 & 23,2 & 22,2 & 21,1 & 20,0 & 19,3 & 18,9 & 17,2 & 17,0 & 17,3 & 18,0 & 20,6 & & \\
\hline Proteína Bruta & 110 & 7 & & & & & & & & & & & \multirow{4}{*}{\multicolumn{2}{|c|}{ 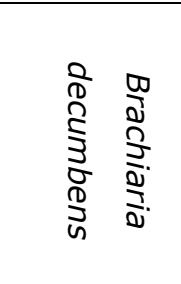 }} \\
\hline$\%$ da MS & 11,9 & 7,7 & 1,5 & 8,1 & , 0 & 5,1 & 6,5 & 1,4 & 8,4 & 9,6 & 9,0 & 10 & & \\
\hline NDT & 59,1 & 56,6 & 55,0 & 54,0 & 49,3 & 47,1 & 50,5 & 52,8 & 47,8 & 56,6 & 63,6 & 61,4 & & \\
\hline Disponibilidade & 22,7 & 22,2 & 21,1 & 20,0 & 19,3 & 18,6 & 17,9 & 17,4 & 17,0 & 18,0 & 18,8 & 23,2 & & \\
\hline Proteína Bruta & & & & & & & & & & & & & \multirow{4}{*}{\multicolumn{2}{|c|}{ 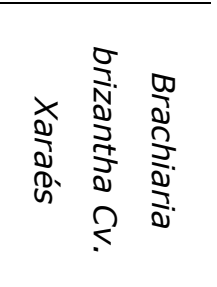 }} \\
\hline$\%$ da MS & כונד & ك, & 10,1 & 5,0 & دار & 0,7 & ك & 1,1 & & 11,2 & (1) & & & \\
\hline NDT & 61,2 & 58,2 & 56,7 & 55,5 & 54,0 & 53,5 & 49,4 & 51,2 & 54,3 & 56,1 & 58,5 & 59,3 & & \\
\hline Disponibilidade & 23,2 & 22,9 & 22,5 & 21,8 & 20,0 & 19,3 & 17,8 & 17,2 & 17,5 & 17,5 & 18,0 & 20,0 & & \\
\hline Proteína Bruta & 123 & 8 & 02 & 0 & 78 & 61 & 71 & 8 & 80 & & ר & & \multirow{4}{*}{\multicolumn{2}{|c|}{ 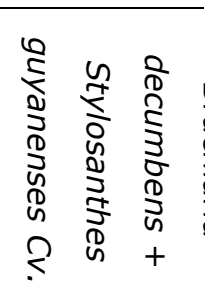 }} \\
\hline$\%$ da MS & IL, & ( & נ, כ, & (1) & 1,0 & 0,1 & 1,4 & 0,2 & 0,7 & 10,4 & 11,0 & $\vdots$ & & \\
\hline NDT & 59,6 & 57,9 & 56,9 & 55,3 & 51,0 & 48,6 & 52,0 & 54,1 & 49,3 & 57,1 & 64,3 & $62,4 \underset{\frac{1}{J}}{\stackrel{D}{J}}$ & & \\
\hline Disponibilidade & 22,5 & 22,3 & 21,9 & 21,1 & 20,0 & 19,3 & 19,3 & 17,5 & 17,0 & 17,0 & 18,1 & 21,5 & & \\
\hline
\end{tabular}


PAULA, E.J.H. et al. Simulação do ganho em produtividade e associação entre características de crescimento e reprodutivas em um rebanho Nelore submetido à seleção. PUBVET, Londrina, V. 6, N. 30, Ed. 217, Art. 1446, 2012.

TABELA 3 - Valor nutricional, consumo e período de utilização do suplemento protéico por categoria animal durante a seca.

\begin{tabular}{lccccc}
\hline \multirow{2}{*}{ Lotes de manejo } & $\begin{array}{c}\text { Consumo } \\
\text { em }\end{array}$ & & \multicolumn{2}{c}{ Valor Nutricional } & Período de utilização \\
\cline { 3 - 6 } & $\begin{array}{c}\text { quilogramas } \\
(\mathrm{kg})\end{array}$ & & & & \\
& & & & & \\
\hline Vacas multíparas & 0,250 & 46 & NDT $(\%)$ & Início & Fim \\
Vacas primíparas & 0,250 & 46 & 34,1 & $1 /$ jul & $1 /$ out \\
Bezerros & 0,080 & 16 & 70,0 & $11 /$ jul & $1 /$ out \\
Novilhas & 0,150 & 46 & 34,1 & $1 /$ jul & 1 /out \\
Touros & 0,300 & 46 & 34,1 & $1 /$ jul & $1 /$ out \\
\hline
\end{tabular}

\section{RESULTADOS E DISCUSSÃO}

Considerando a característica P205 durante 20 anos de simulação para o rebanho em questão, observou-se que nos machos esta característica apresentou variação significativa $(P<0,01)$, passou de $205 \mathrm{~kg}$ no sexto ano de simulação, para $222 \mathrm{~kg}$ no último ano simulado, resultando em um adicional de $8,2 \%$ no peso vivo dos bezerros aos 205 dias, conforme pode ser observado na Figura 1. A padronização dos pesos corporais é de suma importância para que a comparação dos desempenhos dos animais seja possível (LÔBO \& MARTINS FILHO, 2002).

As fêmeas também apresentaram ganhos expressivos para P205 que diferiram significativamente $(P<0,01)$ durante os anos simulados, resultando ao final da simulação um adicional de $14 \mathrm{~kg}$ ou 7,5\% (Figura 2). Esse resultado é fruto do ganho genético devido à seleção, pois os touros utilizados no rebanho foram escolhidos de acordo com os critérios de seleção, que contemplou altas taxas de crescimento e habilidade materna, ocasionando uma elevação no peso dos bezerros aos 205 dias. Além disso, o programa 
PAULA, E.J.H. et al. Simulação do ganho em produtividade e associação entre características de crescimento e reprodutivas em um rebanho Nelore submetido à seleção. PUBVET, Londrina, V. 6, N. 30, Ed. 217, Art. 1446, 2012.

considerou o nível nutricional imutável durante os anos simulados, logo a diferença nos pesos é resultado da seleção para a característica econômica contemplada.

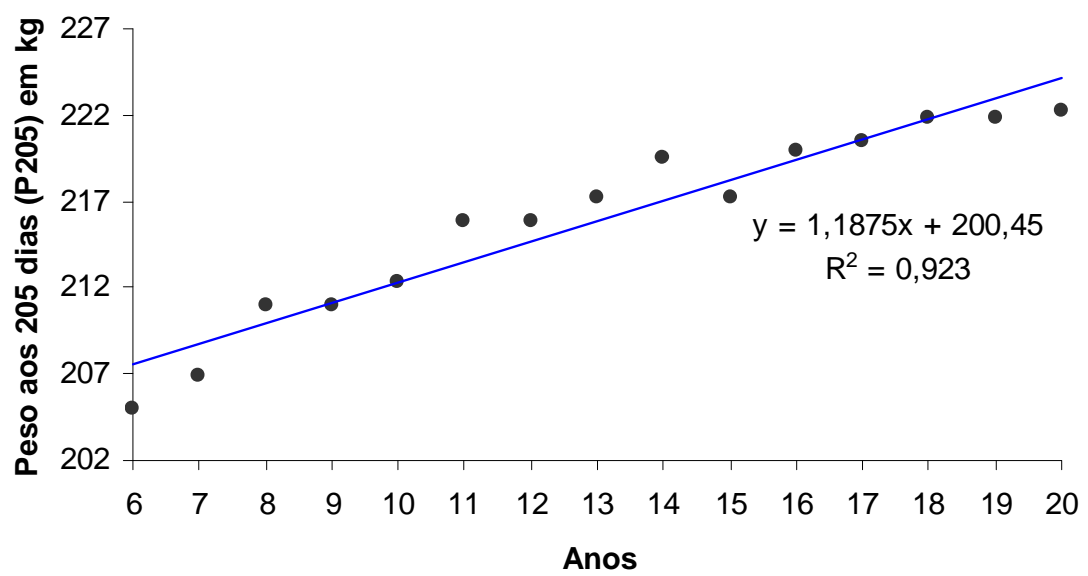

FIGURA 1 - Pesos dos machos ajustados aos 205 dias, simulados por 20 anos em um rebanho seleção de Nelore no Cerrado.

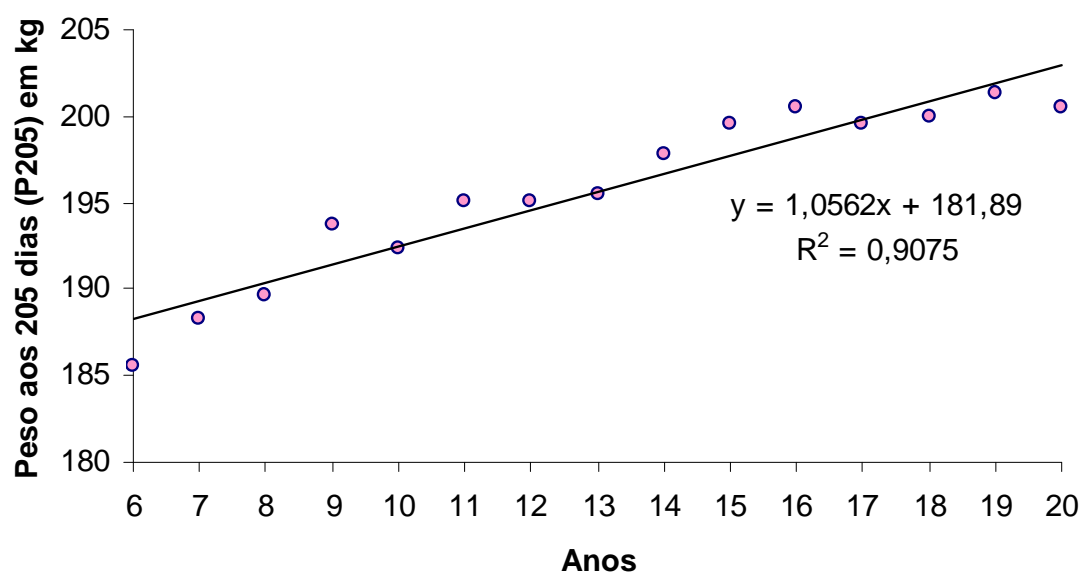

FIGURA 2 - Pesos das fêmeas ajustados aos 205 dias, simulados por 20 anos em um rebanho seleção de Nelore no Cerrado.

BERTAZZO et al. (2004), trabalhando com 56.413 animais da raça Nelore em diferentes regiões do país e sob diferentes condições de criação e manejo, estudaram o peso padronizado aos 205 dias de machos e fêmeas e 
PAULA, E.J.H. et al. Simulação do ganho em produtividade e associação entre características de crescimento e reprodutivas em um rebanho Nelore submetido à seleção. PUBVET, Londrina, V. 6, N. 30, Ed. 217, Art. 1446, 2012.

obtiveram média geral de 171,39 $\pm 30,96 \mathrm{~kg}$, inferiores aos simulados neste estudo.

Os GMDs obtidos da simulação foram de 0,943 e 0,848 $\mathrm{kg}$ para machos e fêmeas, respectivamente (Figuras 3 e 4).

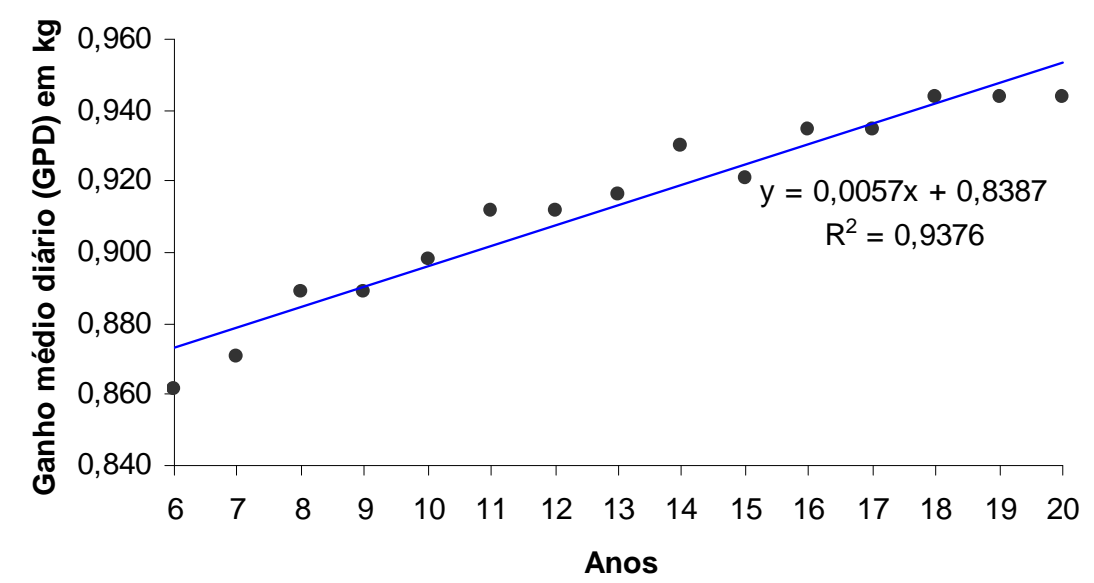

FIGURA 3 - Ganhos médios diários pré-desmame para machos, simulados por 20 anos em um rebanho seleção de Nelore no Cerrado.

Esses resultados quando submetidos à análise de regressão apresentaram variação significativa $(P<0,01)$ e são considerados altos quando comparados com os publicados na literatura para a raça Nelore. VIU et al. (2006), estudando 2.973 bezerros Nelore PO nascidos entre 1992 e 2003, criados em pastagem na região do Cerrado brasileiro, sem suplementação por creep-feeding, relataram GMD pré-desmame de 0,590 \pm 0,09 $\mathrm{kg}$ para machos e 0,547 $\pm 0,08 \mathrm{~kg}$ para fêmeas. 


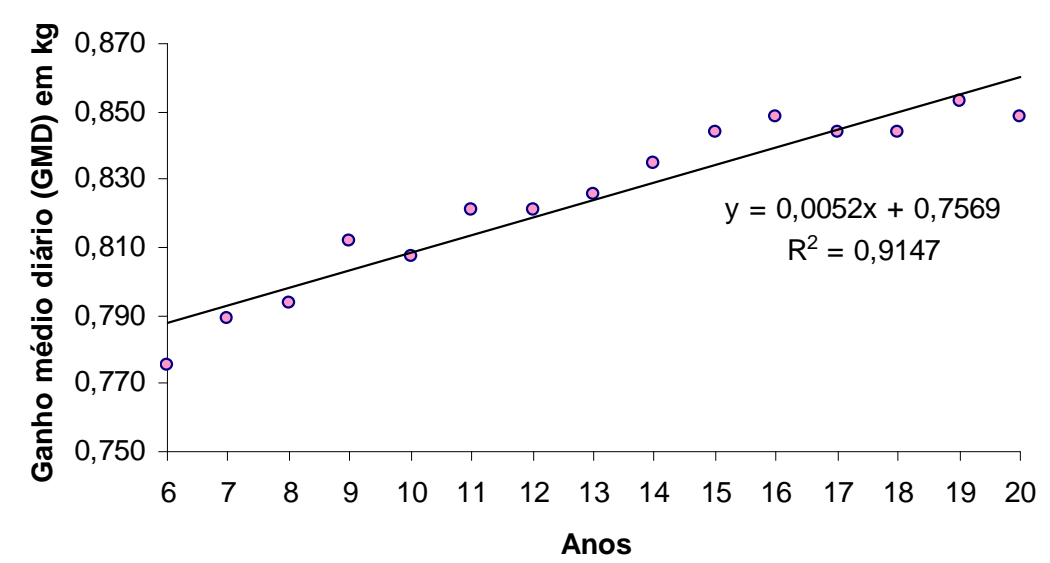

FIGURA 4 - Ganhos médios diários pré-desmame para fêmeas, simulados por 20 anos em um rebanho seleção de Nelore no Cerrado.

Neste estudo, os animais foram produto de gerações consecutivas de seleção para crescimento, e suas mães também foram selecionadas para habilidade maternal, o que influenciou diretamente o GMD dos bezerros, principalmente nos 120 dias iniciais. Além do criterioso diferencial de seleção, houve também o melhoramento ambiental, pois os bezerros tiveram acesso ao creep-feeding durante todo o período de aleitamento, o que ajuda a explicar os altos desempenhos.

Segundo MUNIZ et al. (2005), o desempenho do bezerro até a desmama é conseqüência de seu potencial para crescimento e da habilidade materna de sua mãe. A produção de leite é o principal fator no desenvolvimento do bezerro, tendo coeficiente de correlação de $+0,6$, em média, entre produção de leite da vaca e peso do bezerro à desmama.

Com relação ao peso maduro (PM), nesta simulação, o mesmo passou de $396 \mathrm{~kg}$ no sexto ano para $465 \mathrm{~kg}$ no último (Figura 5). A variação das médias de PM obtidas na simulação quando submetidas à análise de regressão foi significativa $(P<0,01)$. 


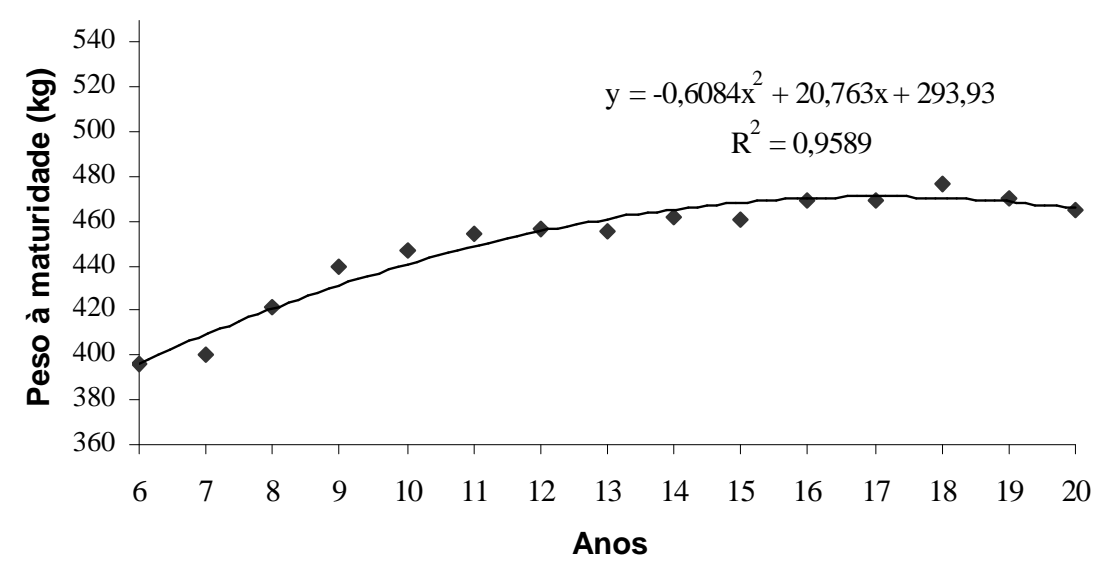

FIGURA 5 - Evolução da média do peso à maturidade do sexto ao vigésimo ano de simulação no rebanho BRGN.

Esses resultados concordam com dados da literatura que demonstram alta correlação entre seleção para alto potencial de crescimento e aumento no PM (McMANUS et al. 2002). FARIA et al. (2008) em estudo das correlações genéticas entre escores visuais e características de crescimento, também observaram maiores pesos a maturidade para animais da raça Nelore selecionados para crescimento.

De acordo com BARBOSA (1991), vários trabalhos têm mostrado que em sistemas de criação extensiva, o maior peso à maturidade está associado com maior incidência de distocia, períodos de gestação mais longos, menores taxas de parição, custos mais altos para manutenção dos rebanhos de vacas, redução da eficiência reprodutiva, intervalos de partos mais longos, maior idade ao primeiro parto e menor taxa de concepção durante a vida útil da vaca. Porém, MERCADANTE et al. (2002) trabalharam com o rebanho seleção Nelore do Instituto de Zootecnia (IZ) e chegaram à conclusão que a seleção para crescimento não influenciou no desempenho reprodutivo das fêmeas apesar de ter aumentado o PM.

No entanto, MARSHALL et al., (1983) e MARSHALL et al., (1987) verificaram que a relação entre o número de bezerros desmamados e o peso das vacas não era linear, diminuindo a partir de um determinado peso das 
vacas. Por outro lado, a quantidade de energia metabolizável consumida pelas vacas durante a vida útil, apresentou relação linear com o peso à maturidade das mesmas. Estes resultados indicam que existe um peso ótimo das vacas quanto à eficiência da produção com base no peso à desmama dos bezerros. Assim, as decisões sobre qual deve ser a variação aceitável do tamanho das vacas podem variar de acordo com os recursos genéticos e ambientais disponíveis, bem como com os custos de manutenção dos diferentes sistemas de produção de bovinos de corte.

Na Figura 6 pode-se observar que a condição corporal das vacas ao parto (CVP) decaiu durante os primeiros treze anos de simulação, passando de 4,7 para 4,4, com perda de 9,3 pontos percentuais, tendendo a estabilidade nos últimos anos simulados; lembrando que o modelo DECI utiliza a escala de um a nove pontos, descrita por HERD \& SPROT (1996). Ainda de acordo com esses autores, neste método de avaliação da condição corporal, escores variando entre quatro e oito pontos seriam desejáveis por não afetarem negativamente a reprodução do rebanho.

Ao analisar e comparar as médias pelo teste de $\mathrm{F}$, a CVP apresentou significância ao nível de $1 \%(P<0,01)$.

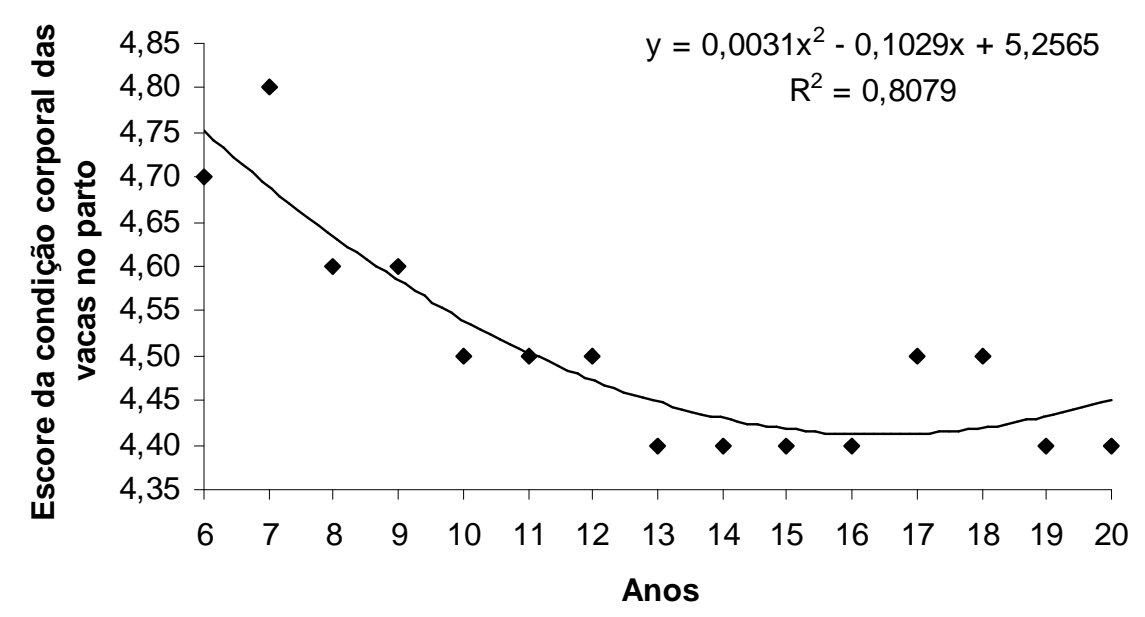

FIGURA 6 - Escores da condição corporal das vacas na ocorrência do parto, simulados por 20 anos em um rebanho seleção de Nelore no Cerrado. 
Segundo SILVA et al. (2005), a condição corporal da fêmea logo após o parto influencia o período da involução uterina, que determina o retorno da atividade ovariana. Os autores ressaltaram que nesta fase a fêmea está em balanço energético negativo, e a boa condição corporal é condição essencial para aumentar a eficiência reprodutiva do rebanho.

BARBOSA (1991) verificou correlação genética desfavorável entre peso em várias idades e características de eficiência reprodutiva em fêmeas bovinas de corte, sugerindo que a seleção para peso pode resultar em redução na eficiência produtiva total dos rebanhos.

A produção de leite das vacas Nelore PO da Embrapa Cerrados, após 20 anos de simulação está apresentada na Figura 7. Houve, com o passar dos anos, acréscimo linear de $5,5 \%$ na produção de leite $(P<0,01)$, chegando à média de $6,17 \mathrm{~kg} / \mathrm{vaca} / \mathrm{dia}$. Esses resultados suportam os altos desempenhos dos bezerros tanto em GMD quanto em P205.

Segundo McMANUS et al. (2002), a habilidade materna é um conjunto de atributos que a vaca deve possuir para fornecer o melhor desenvolvimento possível à sua cria, incluindo imunidade passiva, atenção, proteção e capacidade genética de adaptação. Porém, o atributo que mais se destaca é a produção de leite.

No estudo de RIBEIRO \& RESTLE (1991) a produção de leite foi responsável por $56 \%$ da variação do peso do bezerro à desmama. 
PAULA, E.J.H. et al. Simulação do ganho em produtividade e associação entre características de crescimento e reprodutivas em um rebanho Nelore submetido à seleção. PUBVET, Londrina, V. 6, N. 30, Ed. 217, Art. 1446, 2012.

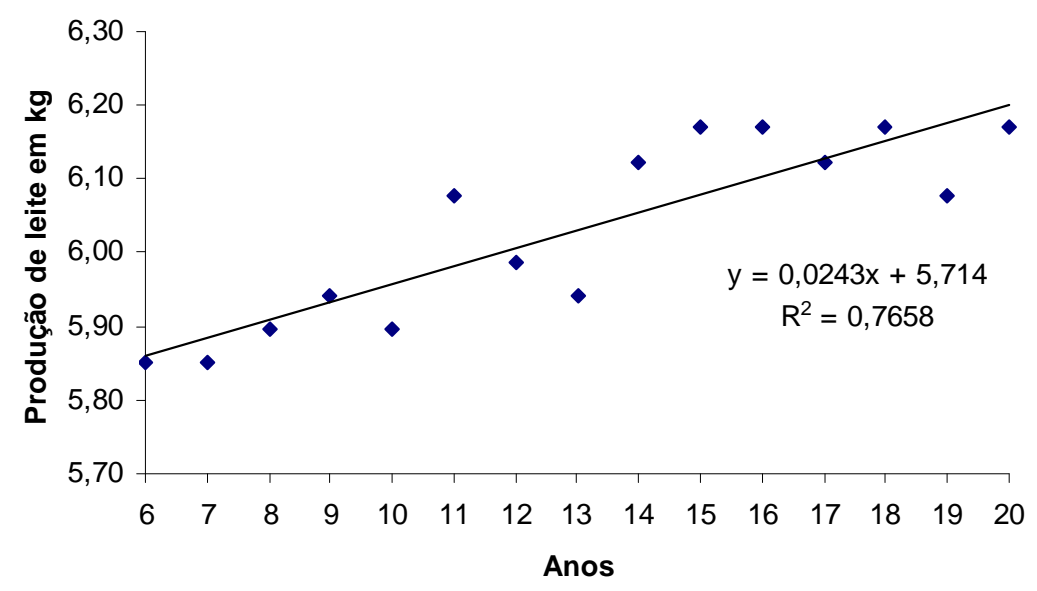

FIGURA 7 - Produções de leite das vacas, simuladas por 20 anos do rebanho seleção de Nelore da Embrapa Cerrados.

Na Figura 8 estão apresentadas as médias de idade à puberdade do sexto ao vigésimo ano de simulação, não havendo variação significativa $(P=0,2742)$. No final de 20 anos de simulação o programa considerou que as novilhas entraram em puberdade com 433 dias de idade, ou 14,4 meses. A literatura relata idades à puberdade para fêmeas Nelore muito superiores às verificadas neste estudo.

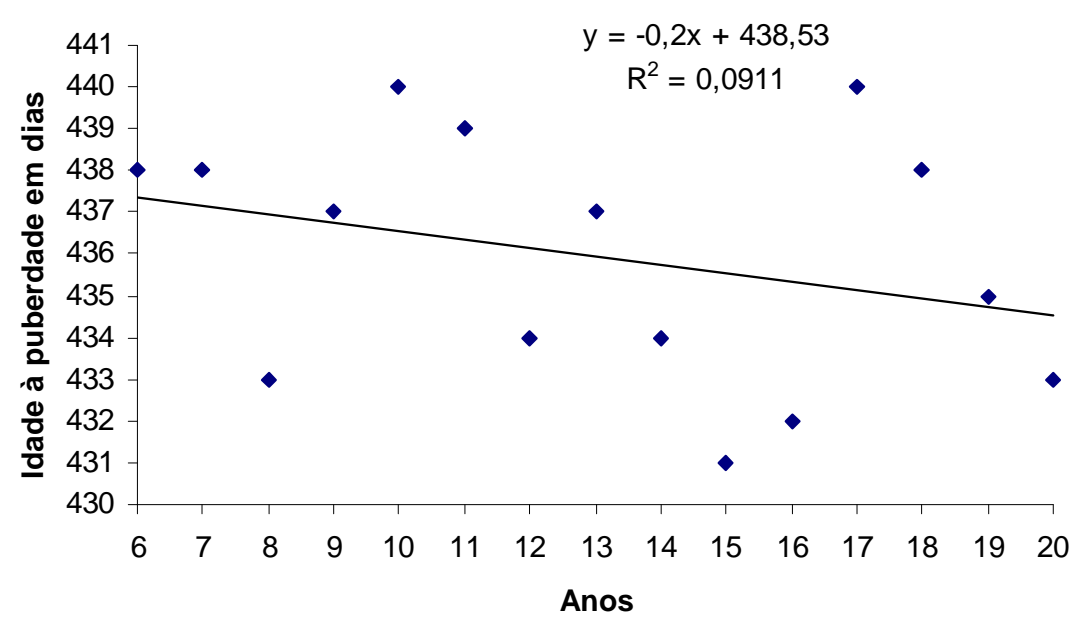

FIGURA 8 - Idades médias à puberdade do rebanho Nelore BRGN em dias, simuladas por 20 anos. 
RESTLE et al. (1999), avaliando o desempenho reprodutivo de fêmeas das raças Charolês, Nelore e suas cruzas recíprocas em um sistema de produção de dois anos, encontraram idade e peso à puberdade de $326 \mathrm{~kg} \mathrm{e}$ 689 dias para novilhas puras Nelore e $346 \mathrm{~kg}$ e 600 dias para novilhas cruzadas, respectivamente. Os autores relataram que reduzir a idade do primeiro acasalamento para dois anos é fácil de ser atingido por meio do uso de pastagens cultivadas. No entanto, reduzir a idade de acasalamento para 13,5 meses requer o uso de suplementação energética associada à pastagem.

Conforme FRIZZO et al. (2003), quando as exigências nutricionais pósdesmama são atendidas, há redução da idade à puberdade e ao primeiro serviço na maioria das novilhas. FRIZZO et al. (2003), trabalharam com fêmeas Hereford de cruzas com Nelore em diferentes sistemas alimentares na pós-desmama, e observaram que o primeiro estro foi manifestado aos 439 dias, com o animal pesando $263 \mathrm{~kg}$.

Em se tratando de idade ao primeiro parto (IPP), nesta simulação o mesmo foi de 741 dias (Figura 9), o que corresponde a 24,7 meses de idade, indicando que as fêmeas tornaram-se prenhas com 14,7 meses. Esses resultados são considerados ótimos em relação aos encontrados na literatura. Porém, ao comparar as médias pelo teste de $\mathrm{F}$ a variação não foi significativa $(P=0,8273)$, indicando que a seleção para crescimento não influenciou na IPP.

De acordo com GRESSLER (2004), a IPP de rebanhos da raça Nelore possui média aproximada de 37,3 meses, sendo que as estimativas de herdabilidade variam entre 0 a $0,38 \mathrm{em}$ diversos modelos utilizados. Estimativas de herdabilidade em novilhas desafiadas precocemente aos 14 meses indicaram que a característica possui variabilidade genética suficiente para ser considerada como critério de seleção para precocidade sexual de reprodutores da raça Nelore. 
PAULA, E.J.H. et al. Simulação do ganho em produtividade e associação entre características de crescimento e reprodutivas em um rebanho Nelore submetido à seleção. PUBVET, Londrina, V. 6, N. 30, Ed. 217, Art. 1446, 2012.

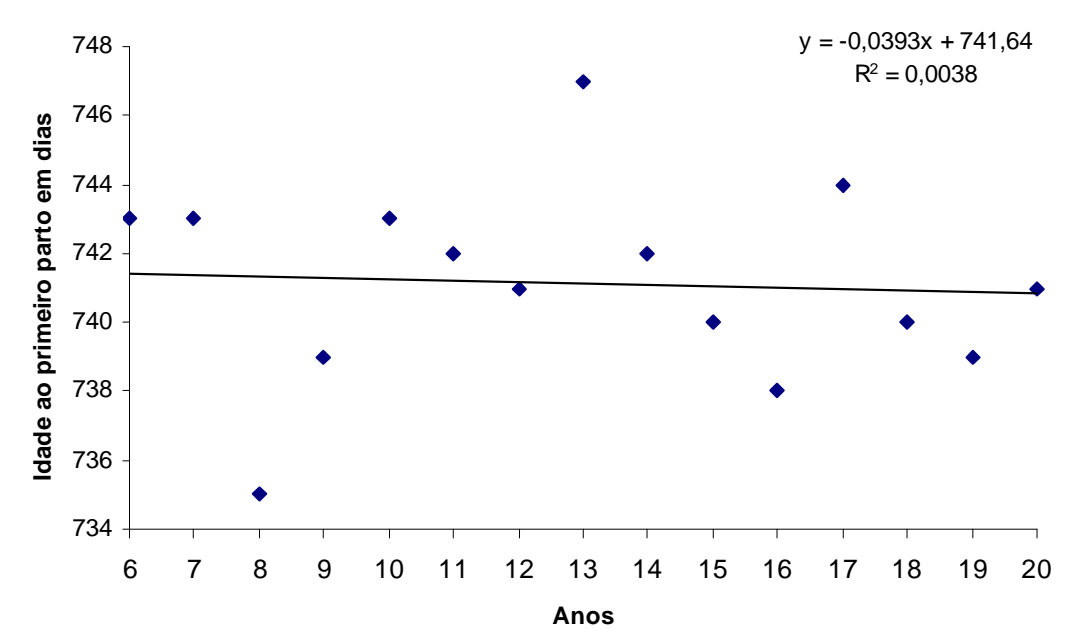

FIGURA 9 - Média de idade ao primeiro parto das novilhas Nelore, em dias, durante a simulação.

Nesta simulação o PS aumentou em 5,4\% ao longo dos anos, chegando a uma média de 78 dias de intervalo parto-concepção (Figura 10), porém a comparação entre as médias não apresentou variação significativa $(P=0,3437)$. Esses resultados são considerados muito baixos, o que é altamente desejado.

Segundo AZEVÊDO et al. (2006), a duração ideal do PS é de 60 a 90 dias, considerando-se a meta de produção de um bezerro por ano. Entretanto, animais criados em regiões tropicais apresentam PS excessivamente longo.

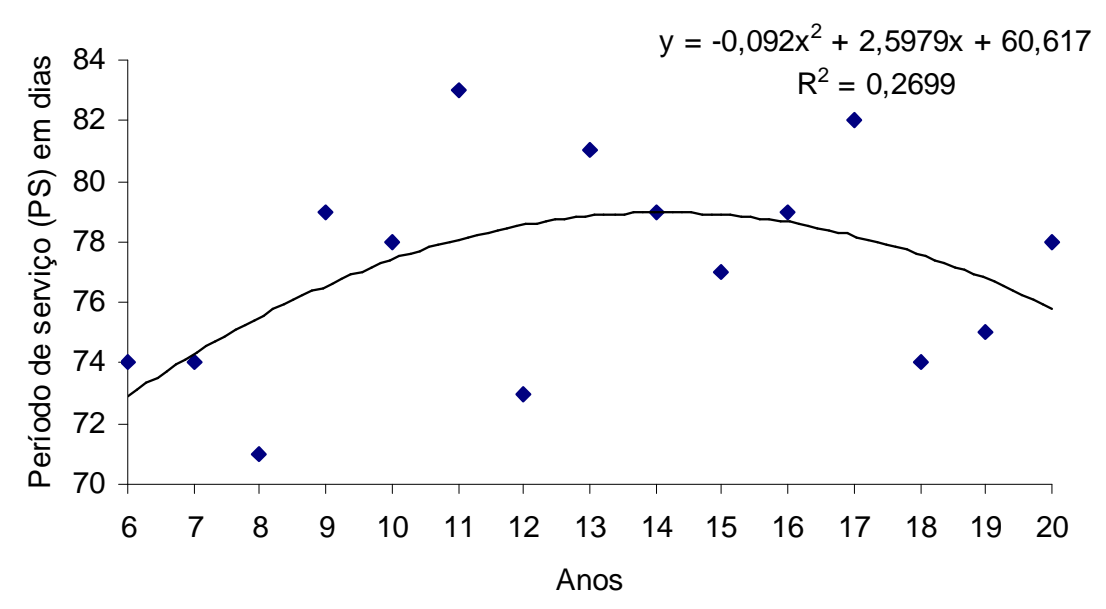

FIGURA 10 - Período médio de serviço, em dias, durante os anos simulados. 
CAVALCANTE et al. (2001), analisaram 587 dados relativos aos períodos de serviço de animais da raça Nelore criados extensivamente na Amazônia Oriental, e chegaram ao PS médio de 146,51 \pm 43,05 dias (4,82 meses), concluindo também que o PS foi influenciado pelo efeito pai da vaca.

Analisando 10.069 fêmeas da raça Nelore, participantes do PMGRN, AZEVEDO et al. (2006) estimaram o PS e obtiveram média de $165,76 \pm$ 110,29 dias.

A taxa de prenhez (TP) do rebanho simulado, mesmo sendo selecionado para crescimento, com aumento linear dos pesos à maturidade, praticamente permaneceu inalterada durante o período de simulação e desta forma não apresentou variação significativa $(P=0,7118)$ como pode ser observado na Figura 11 . No último ano $77 \%$ das fêmeas expostas aos touros tornaram-se gestantes, contradizendo resultados da literatura que relatam correlações altas de maior peso da matriz com menor desempenho reprodutivo (BARBOSA, 1991).

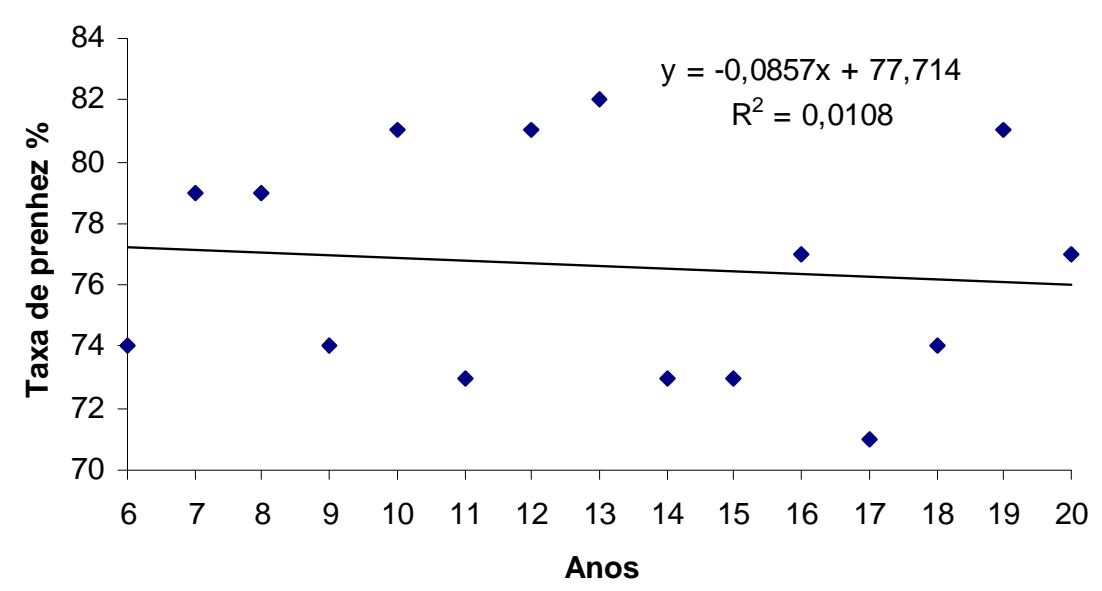

FIGURA 11 - Evolução da taxa de prenhez do rebanho Nelore, simulada por 20 anos.

Resultados inferiores $(43,2 \%)$ foram obtidos por GRECELLÉ et al. (2006) ao trabalharem com 117 vacas com cria ao pé, da raça Nelore e cruzas com 
Hereford, em pastagens de Brachiaria no estado do Paraná. ALMEIDA et al. (2002) também relataram TP de 40 e 51\% para os anos de 1999 e 2000 para um rebanho de 93 vacas mestiças (Bos taurus $\times$ Bos indicus) em pastagens nativas de Andropógon.

Índices superiores $(82 \pm 7 \%)$ de TP foram relatados por MERCADANTE (2006), que avaliou a resposta da seleção para características de crescimento em fêmeas Nelore do IZ. A autora relatou TP de novilhas colocadas em reprodução aos 24 meses de $62 \pm 9 \%$, e concluiu que a seleção para maiores pesos não acarretou diferenças no desempenho reprodutivo das fêmeas.

Portanto, a seleção para alto potencial de crescimento e habilidade materna, pela utilização de touros com DEPs apropriadas, proporcionou, durante os 20 anos de simulação, melhorias no rebanho seleção Nelore da Embrapa Cerrados. A seleção para habilidade materna teve como resposta direta aumento de 5,5\% na produção de leite, acarretando maior ganho de peso pré-desmama $(9,3 \%)$ e aumento do P205 (8,2\%). Com maiores ganhos de peso, os animais atingiram a puberdade precocemente (14,4 meses), diminuindo o custo na produção de fêmeas de reposição, encurtando o intervalo de gerações e aumentando o ganho genético. Porém, foram verificadas maiores médias para PM $(465 \mathrm{~kg})$, indicando maiores custos de manutenção do rebanho devido ao aumento no tamanho corporal e, concomitantemente, nas exigências de mantença desses animais.

A maior produção de leite das mães acarretou diminuição na condição corporal ao parto $(9,3 \%)$ e aumentou o PS $(5,4 \%)$. Porém, a taxa de prenhez do rebanho praticamente permaneceu inalterada (77\%), demonstrando que, apesar da queda na condição corporal, não houve prejuízo no que se refere ao desempenho reprodutivo do rebanho. Durante a simulação não foram realizadas mudanças no sistema nutricional fornecido aos animais e este mostrou-se capaz de aportar os requerimentos dos animais selecionados, teoricamente, mais exigentes nutricionalmente. 
PAULA, E.J.H. et al. Simulação do ganho em produtividade e associação entre características de crescimento e reprodutivas em um rebanho Nelore submetido à seleção. PUBVET, Londrina, V. 6, N. 30, Ed. 217, Art. 1446, 2012.

\section{CONCLUSÃO}

Nas condições simuladas, a seleção para crescimento e habilidade materna propiciou incremento nos índices de produção do sistema extensivo de criação de Nelore da Embrapa Cerrados no horizonte de vinte anos. Além disso, o sistema nutricional (forragens e suplementação) forneceu o aporte necessário de nutrientes permitindo que os animais demonstrassem resposta aos critérios de seleção utilizados.

O modelo de simulação DECI foi capaz de predizer as respostas à seleção genética para altas taxa de crescimento e habilidade materna demonstrando que o modelo prediz as interações biológicas do animal e do sistema de produção.

Apesar dos critérios de seleção adotados terem aumentado o peso aos 205 dias, o ganho médio diário pré-desmame e o peso à maturidade, estes não interferiram negativamente na idade a puberdade, período de serviço, idade ao primeiro parto e na taxa de prenhez do rebanho.

\section{AGRADECIMENTOS}

Aos pesquisadores da Embrapa Cerrados que cederam as informações básicas para que este trabalho fosse realizado. A CAPES pela concessão da bolsa de estudos durante o mestrado. Aos estudantes de pós-graduação do setor de Produção Animal da Universidade Federal de Goiás.

\section{REFERÊNCIAS}

ALMEIDA, L. S. P. de; LOBATO, J. F. P.; SCHENKEL, F. S. Data de desmame e desempenho reprodutivo de vacas de corte. Revista Brasileira de Zootecnia. V. 31, n. 3, p. 1223-1229, 2002.

AZEVÊDO, D. M. M. R.; MARTINS FILHO, R.; LÔBO, R. N. B.; MALHADO, C. H. M.; LÔBO, R. B.; MOURA, A. de A. A.; PIMENTA FILHO, E. C. Desempenho reprodutivo de vacas Nelore no Norte e Nordeste do Brasil. Revista Brasileira de Zootecnia. v.35, n.3, p. 988-996, 2006. 
BARBOSA, P. F. Análise genético-quantitativa de características de crescimento e reprodução em fêmeas da raça Canchim. Ribeirão Preto, 1991, 237p. Tese (Doutorado em Ciências) - Universidade de São Paulo. 1991.

BERTAZZO, R. P.; FREITAS, R. T. F.; GONÇALVES, T. M. Parâmetros genéticos de longevidade e produtividade de fêmeas da raça Nelore. Revista Brasileira de Zootecnia. v. 33, n. 5, p. 1118-1127, 2004.

CAVALCANTE, F. A.; MARTINS FILHO, R.; CAMPELLO, C. C.; LOBO, R. N. B.; MARTINS, G. A. Período de serviço em rebanho Nelore na Amazônia Oriental. Revista Brasileira de Zootecnia. v. 30, n. 5, p. 1456-1459, 2001.

FARIA, C. U.; MAGNABOSCO, C. U.; AlbuQUeRQUE, L. G.; REYES, A. BeZERRA, L. A. F.; LÔBO, R. B. Estimativas das correlações genéticas entre escores visuais e características de crescimento em bovinos da raça Nelore utilizando modelos bayesianos linear-limiar. Ciência Animal Brasileira. v. 9, p. 327-340, 2008.

FRIZZO, A.; ROCHA, M. G. da; RESTLE, J.; MONTAGNER, D. B.; FREITAS, F. K. de; SANTOS, D. T. dos. Suplementação energética na recria de bezerras de corte mantidas em pastagem de inverno. Revista Brasileira de Zootecnia. v. 32, n. 3, p. 643-652, 2003.

GRECELlÉ, R. A.; BARCELlos, J. O. J.; BRACCINI NETO, J.; COSTA, E. C. da; PRATES, Ê, R. Taxa de prenhez de vacas Nelore $X$ Hereford em ambiente subtropical sob restrição alimentar. Revista Brasileira de Zootecnia. v. 35, n. 4, p. 1423-1430, 2006.

GRESSLER, S. L. Fatores ambientais e genéticos do perímetro escrotal e da idade ao primeiro parto de novilhas Nelore desafiadas tradicional ou precocemente. 2004. Tese (Doutorado em Ciência Animal) - Faculdade Federal de Minas Gerais - Belo Horizonte. 2004.

HERD, D. B.; SPROTT, L. R. Body condition, nutrition and reproduction of beef cow. Texas Agricultural Extention Service Bulletin. Texas, p. 3-11, 1996.

LÔBO, R. N. B.; MARTINS FILHO, R. Avaliação de métodos de padronização dos pesos corporais às idades de 205, 365 e 550 dias. Revista Brasileira de Zootecnia. v. 31, n. 4, p. 1695-1706, 2002.

MAGNABOSCO, C. de U. Avaliação da eficiência de produção em um rebanho de seleção da raça Nelore no Cerrado: um estudo de simulação usando o DECI - Decision Evaluator for the Cattle Industry. Ed. Embrapa Cerrados, Relatório de Estágio Pós Doutoral, 32p. 2004.

MAGNABOSCO, C. de U.; BARIONI, L. G.; PAULINO, P. V. R.; WILLIAMS, C. B.; SAUERESSIG, M.; FARIA, C. U.; MARTHA JUNIOR, G. B.; LÔBO, R. B.; PASCOA, L.; SAINZ, R. D. Análise de decisão considerando diferentes méritos genéticos para touros em um rebanho da raça Nelore utilizando o programa DECI. In. $43^{\circ}$ REUNIÃO ANUAL DA SOCIEDADE BRASILEIRA DE ZOOTECNIA, 2006, João Pessoa. Anais... João Pessoa: SBZ/UFPB, 2006. CD ROM.

MARSHALL, T. E.; STEWART, T. S.; MARTIN, T. G. Optimal mature size of Angus cows for maximum cow productivity. In: INDIANA ASSOCIATION ANNUAL CONVENTION, 10. 1983 West Lafayette, Proceedings, p. 43-48, 1983.

MARSHALL, T. E.; MOHLER, M. A.; STEWART, T. S. Relationship of lifetime productivity with mature weight and maturation rate in Red Poll cows. Animal Production, v. 39, n. 3, p. 383$387,1987$. 
McMANUS, C.; SAUERESSig, M. G.; FALCÃO, R. A.; SERRANO, G.; MARCELINHO, K. R. A.; PALUDO, G. R. Componentes reprodutivos e produtivos no rebanho de corte da Embrapa Cerrados. Revista Brasileira de Zootecnia, v. 31, n. 2, p. 648-657, 2002.

MEIRELLES, F. D. P. Modelo computacional de um rebanho bovino de corte virtual utilizando Simulação de Monte Carlo e Redes Neurais Artificiais. 2005. 105f. Tese (Doutorado em Qualidade e Produtividade Animal) - Faculdade de Zootecnia e Tecnologia de Alimentos, Universidade de São Paulo, Pirassununga. 2005.

MERCADANTE, M. E. Z.; PACKER, I. U.; RAZOOK, A. G.; CYRILLO, J. N. dos S. G.; FIGUEIREDO, L. A. de. Dias ao parto de fêmeas Nelore de um experimento de seleção para crescimento. I - Modelo de Repetibilidade. Revista Brasileira de Zootecnia. V. 31, n.4, p. 1715-1725, 2002.

MERCADANTE, M. E. Crescimento e reprodução. Disponível em www.iz.sp.gov.br/artigos/documentos/Mercadante . 2006. Acesso em 10 de março de 2007.

MUNIZ, C. A. de S. D; CARVAlHeIRO, R.; FRIES, L. A.; QUEIROZ, S. A. de. Dois critérios de seleção na pré-desmama em bovinos da raça Gir. 1. Estimativas de parâmetros genéticos. Revista Brasileira de Zootecnia. v. 34, n.3, p. 807-815, 2005.

PASCOA, L.; MAGNABOSCO, C. de U.; BARIONI, L. G.; SAUERESSIG, M.; PAULINO, P. V. R.; MARTHA JUNIOR, G. B.; PAULA, E. J. H.; WILLIAMS, C. B.; SAINZ, R. D. Utilização do simulador DECI para avaliar eficiência de seleção para potencial de crescimento em um rebanho Nelore no Cerrado. In. $43^{\circ}$ REUNIÃO ANUAL DA SOCIEDADE BRASILEIRA DE ZOOTECNIA, 2006, João Pessoa. Anais... João Pessoa: SBZ/UFPB, 2006. v. 35.

PEGDEN, D. C.; SHANNON, R. E.; SADOWSKI, R. P. Introduction to simulation using SIMAN, Mc-Graw Hill, 1990, USA, $2^{\circ}$ ed. 1995. 600 p..

RESTLE, J.; POLLI, V. A.; SENNA, D. B. Efeito de grupo genético e heterose sobre a idade à puberdade e sobre o desempenho reprodutivo de novilhas de corte. Pesquisa Agropecuária Brasileira. v. 34, n. 4, p. 701-707, 1999.

RIBEIRO, E. L. A.; RESTLE, J. Desempenho de terneiros Charolês e Aberdeen Angus puros e seus mestiços com Nelore. Pesquisa Agropecuária Brasileira, v. 26, n. 8, p. 1145-1151, 1991.

SAINZ, R. D., BALDWIN, R. L., VERNAZZA, R. F. Models of cattle growth, digestion and lactation. In: INTERNATIONAL WORKSHOP ON SIMULATION MODELS OF TROPICAL MEAT AND MILK PRODUCTION SYSTEMS. Procedings... 2000, Bogotá: 27-30 June, 2000.

SILVA, N. P.; OLIVEIRA FILHO, B. D.; GAMBARINI, M. L. Eficiência reprodutiva de primíparas suplementadas com fósforo, proteína e energia no pré e pós-parto. In: REUNIÃO ANUAL DA SOCIEDADE BRASILEIRA DE ZOOTECNIA, 42., 2005, Goiânia. Anais... Goiânia: Universidade Federal de Goiás, 2005. [CD-ROM].

STATISTICAL ANALYSIS SYSTEMS - SAS. User's guide: Version 6, Cary: 1997. v. 2, 1052p.

TESS, M. W.; KOLSTAD, B. W. Simulation of cow-calf production systems in a range environment: I Model development. Journal Animal Science, v. 78: p.1159-1169, 2000a.

TESS, M. W.; KOLSTAD, B. W. Simulation of cow-calf production systems in a range environment: II Model evolution. Journal Animal Science, v. 78: p.1170-1180, 2000b. 
VIEIRA, A.; LOBATO, J. F. P.; CORRÊA, E. S.; TORRES JÚNIOR, R. A. de A.; COSTA, F. P. Desenvolvimento e desempenho reprodutivo de novilhas Nelore criadas a pasto nos cerrados do centro-oeste brasileiro. Revista Brasileira de Zootecnia. v. 35, n.1, p. 186-192, 2006.

VIU, M. A. de O.; BRASIL, LOPES, D. T.; GAMBARINI, M. L.; OLIVEIRA FILHO, B. D. de; FERRAZ, H. T.; MAGNABOSCO, C. de U.; VIU, A. F. M. Efeito da época do parto, idade materna e sexo sobre o desempenho pré-desmama de bezerros Nelore (Bos taurus indicus), criados extensivamente no Centro-Oeste do Brasil. Archives of Veterinary Science, v.11, n. 3, p. 75-79, 2006.

WILLIAMS, C. B.; BENNETT, G. L.; KEELE, J. W. Simulated influence of post weaning production system on performance of different biological types of cattle: I. Estimation of model parameters. Journal Animal Science, v. 73, p. 665 - 673, 1995a.

WILLIAMS, C. B.; BENNETT, G. L.; KEELE, J. W. Simulated influence of post weaning production system on performance of different biological types of cattle: II. Carcass composition, retail product, and quality. Journal Animal Science, v. 73, p. $674-685$, 1995b.

WILLIAMS, C. B.; BENNETT, G. L.; KEELE, J. W. Simulated influence of post weaning production system on performance of different biological types of cattle: III. Biological Efficiency. Journal Animal Science, v. 73, p. 686 - 698, 1995c.

WILLIAMS, C. B.; BENNETT, G. L.; JENKINS, T. G.; CUNDIFF, L. V.; FERRELL, C. L. Using simulation models to predict feed intake: Phenotypic and genetic relationships between observed and predict values in cattle. In. Journal Animal Science, v. 84, p. 1310-1316, 2006.

WILLIAMS, C.B.; JENKINS, T.G. Decision Evaluator for the Cattle Industry. User's Guide and Software. 2000. Download available. http://www.marc.usda.gov 